\title{
CAIXA: a catalogue of AGN in the XMM-Newton archive
}

\section{Multiwavelength correlations}

\author{
S. Bianchi ${ }^{1,2}$, N. F. Bonilla ${ }^{2}$, M. Guainazzi ${ }^{2}$, G. Matt ${ }^{1}$, and G. Ponti ${ }^{3}$ \\ 1 Dipartimento di Fisica, Università degli Studi Roma Tre, via della Vasca Navale 84, 00146 Roma, Italy \\ e-mail: bianchi@fis.uniroma3.it \\ 2 XMM-Newton Science Operations Center, European Space Astronomy Center, ESA, Apartado 50727, 28080 Madrid, Spain \\ ${ }^{3}$ Laboratoire APC, UMR 7164, 10 rue A. Domon et L. Duquet, 75205 Paris, France
}

Received 20 February 2009 / Accepted 21 April 2009

\section{ABSTRACT}

\begin{abstract}
Aims. The availability of large amounts of multiwavelength data allows us to perform an extensive statistical analysis to look for correlations between different parameters of AGN. The physical implications of these correlations, when considered within the framework of current AGN models, can be enlightening in the resolution of the problems and the open issues which still characterise this class of sources.

Methods. We presented CAIXA, a Catalogue of AGN in the XMM-Newton Archive, in a companion paper. It consists of radio-quiet $\mathrm{X}$-ray unobscured sources, which cover a range in X-ray luminosities between $L_{2-10 \mathrm{keV}}=2.0 \times 10^{41}$ and $3.9 \times 10^{46} \mathrm{erg} \mathrm{s}^{-1}$, and in redshift from $z=0.002$ to $z=4.520$. Here, a systematic search for correlations between the X-ray spectral properties and the multiwavelength data was performed for the sources in CAIXA. All the significant ( $>99.9 \%$ confidence level) correlations are discussed along with their physical implications for current models of AGN.

Results. Two main correlations are discussed in this paper: a) a very strong anti-correlation between the $F W H M$ of the $\mathrm{H} \beta$ optical line and the ratio between the soft and the hard X-ray luminosity. Although similar anti-correlations between optical line width and $\mathrm{X}$-ray spectral steepness have already been discussed in the literature, we consider the formulation we present in this paper as more fundamental, as it links model-independent quantities. Coupled with a strong anti-correlation between the V to hard X-ray flux ratio and the $\mathrm{H} \beta$ FHWM, it supports scenarios for the origin of the soft excess in AGN, which require strong suppression of the hard X-ray emission; b) a strong (and expected) correlation between the X-ray luminosity and the black hole mass. Its slope, flatter than 1, is consistent with Eddington ratio-dependent bolometric corrections. Moreover, we critically review through various statistical tests the role that distance biases play in the strong radio to X-ray luminosity correlation found in CAIXA and elsewhere; we conclude that only complete, unbiased samples should be used to draw observational constraints on the origin of radio emission in radio-quiet AGN.
\end{abstract}

Key words. galaxies: active - galaxies: Seyfert - X-rays: galaxies

\section{Introduction}

It is now well established that active galactic nuclei (AGN) are powered by accretion onto a central supermassive black hole (Lynden-Bell 1969). Different flavours of unification models are quite successful in explaining most of the varied phenomenology of these sources in terms of a fundamental scenario, which explains the characteristic signatures in the spectra of AGN: a thermal disc emitting in the optical and ultraviolet; a corona of hot electrons producing X-ray photons via inverse Compton scattering of the photons from the disc; dusty gas which reprocesses the nuclear emission into infrared emission and into other X-ray spectral components. However, many aspects related to fundamental properties of AGN are still to be fully understood and many competing models have been developed and need to be tested. One fruitful approach to this kind of investigation is to gather as much multiwavelength data as possible on large numbers of sources and perform a systematic statistical analysis looking for correlations between different parameters. Much progress has been made since the first seminal attempts (e.g. Boroson \& Green 1992), thanks to the availability of ever-increasing data archives. In particular, in the last few years large amounts of high-quality X-ray data have become accessible.

We presented CAIXA, a Catalogue of AGN in the XMM-Newton Archive, in a companion paper (Bianchi et al. 2009, B09 from now on). It consists of all the radio-quiet X-ray unobscured $\left(N_{\mathrm{H}}<2 \times 10^{22} \mathrm{~cm}^{-2}\right)$ active galactic nuclei (AGN) observed by XMM-Newton in targeted observations, whose data are public as of March 2007. With its 156 sources, this is the largest catalogue of high signal-to-noise X-ray spectra of AGN. It covers a range in X-ray luminosities between $L_{2-10 \mathrm{keV}}=$ $2.0 \times 10^{41}$ and $3.9 \times 10^{46} \mathrm{erg} \mathrm{s}^{-1}$, and in redshift from $z=0.002$ to $z=4.520$. For each source in CAIXA we have collected observable and physical parameters more directly related to the accretion onto the supermassive black hole $(\mathrm{BH})$, and less affected by contamination by the host galaxy. Therefore, the EPIC pn X-ray data are complemented by multiwavelength data found in the literature: BH masses, Full Width Half Maximum $(F W H M)$ of $\mathrm{H} \beta$, radio and optical fluxes.

We refer the reader to B09 for all the details on the selection of the catalogue, the X-ray data reduction analysis and the coverage of the multiwavelength data. Here we investigate the correlations between the X-ray and the multiwavelength 


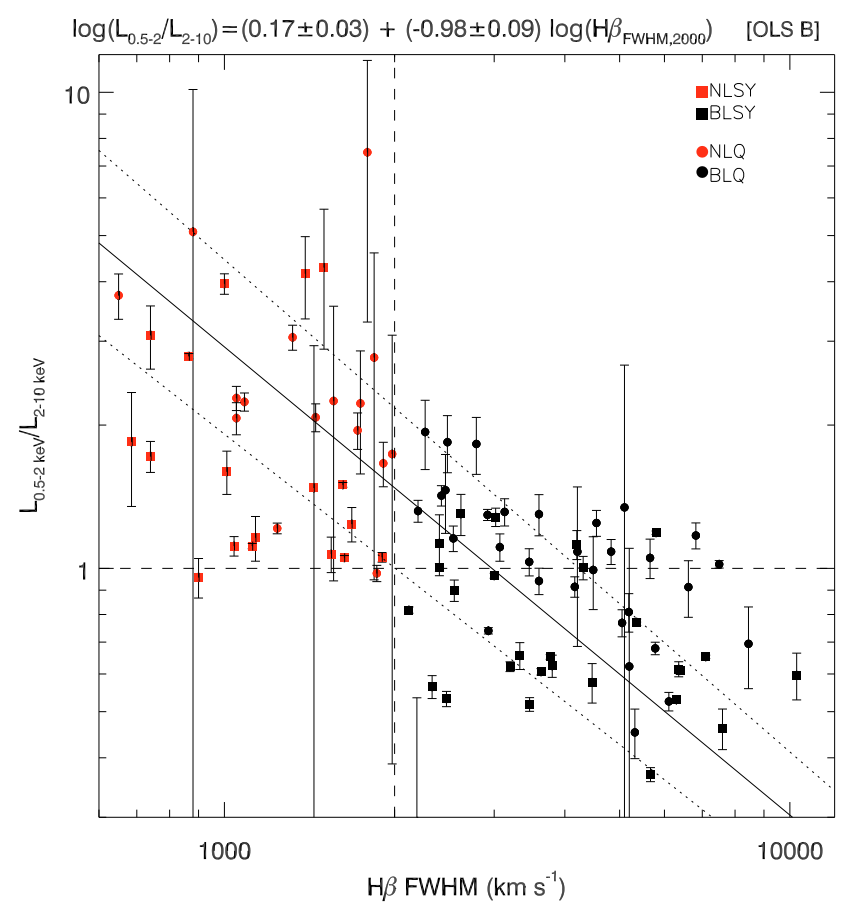

Fig. 1. Left: $0.5-2 / 2-10 \mathrm{keV}$ luminosity ratio vs. $\mathrm{H} \beta F W H M$. The anticorrelation between the two parameters is very significant. The two broken lines represent the $2000 \mathrm{~km} \mathrm{~s}^{-1}$ boundary between broad- and narrow-line objects and $L_{0.5-2} / L_{2-10}=1$. See caption of Fig. 5 for details on the adopted symbols.

properties of the sources in CAIXA. The physical implications of these results will be discussed within the framework of current AGN models, with particular focus on the open issues they still raise.

\section{Results}

We have performed a systematic correlation analysis between all the parameters which characterise the sources in CAIXA. The results are summarized in Table 1 . In this paper, we consider statistically significant only the correlations whose Null Hypothesis Probability (NHP) is lower than $1 \times 10^{-3}$ (corresponding to a confidence level of $99.9 \%$ ), with respect to the Spearman correlation coefficient $\rho$ resulting from a censored fit (see Appendix A for details on this procedure, which takes into account both errors and upper limits on the $Y$ variable).

We will discuss in detail in the next sections all the significant correlations we found in CAIXA.

\subsection{Correlations with $H \beta F W H M$ and the nature of the soft excess}

Looking at Table 1, the most significant correlation with the $F W H M$ of $\mathrm{H} \beta$ is that against the ratio between the soft $(0.5-2 \mathrm{keV})$ and the hard (2-10 keV) X-ray luminosity. The observed anti-correlation is shown in Fig. 1.

Given the unknown origin of the soft excess in AGN, we introduced in B09 this X-ray luminosity ratio as a good parameter to characterize at least the basic property of the soft excess of the sources, i.e. its strength with respect to the primary Xray emission. As already noted in B09, it is very interesting to note that CAIXA does not contain narrow-line objects with a $L_{0.5-2} / L_{2-10}$ ratio lower than 1 and narrow- and broad-line objects are significantly different populations with respect to this parameter. However, just as no dichotomy appears in the optical properties between narrow- and broad-line objects, a smooth transition between high and low X-ray luminosity ratios is also apparent from the data. The best fit for the anti-correlation takes this form:

$$
\log \left(\frac{L_{0.5-2}}{L_{2-10}}\right)=(0.17 \pm 0.03)+(-0.98 \pm 0.09) \log \left(\mathrm{H} \beta_{2000}\right)
$$

where $\mathrm{H} \beta_{2000}$ is the $F W H M$ expressed in units of $2000 \mathrm{~km} \mathrm{~s}^{-1}$. We remind the reader that this correlation, which involves the soft X-ray luminosity, was calculated on the CAIXA subcatalogue which excludes objects with a measured column density in excess of the Galactic one (see B09).

The other correlation with $\mathrm{H} \beta$ found in CAIXA is the one with the hard X-ray spectral slope, confirming previous claims (e.g. Brandt et al. 1997; Piconcelli et al. 2005). An anticorrelation between the $0.1-2.4 \mathrm{keV}$ slope and the $\mathrm{H} \beta$ line width was also presented by Laor et al. (1994) and later confirmed by Boller et al. (1996) and Laor et al. (1997). However, we note here that in CAIXA there is no significant anti-correlation between the $\mathrm{H} \beta$ and the soft $\mathrm{X}$-ray photon index. This is likely another piece of evidence in favour of the fact that the values of $\Gamma$ are very sensitive to the adopted models and it is therefore better to consider the correlations between more model-independent parameters. We therefore believe that our anti-correlation between the X-ray luminosity ratio and $\mathrm{H} \beta$ is a more basic physical correlation, because it links two a priori independent properties of radio-quiet AGN.

The transition from broad- to narrow-line AGN, as well as the strength of the soft excess have been often interpreted as being due to a more fundamental physical parameter, such as the accretion rate or the BH mass (e.g. Laor et al. 1997). However, there is no significant correlation in CAIXA between $L_{0.5-2} / L_{2-10}$ and the Eddington ratio, the $\mathrm{BH}$ mass or any parameter considered in this work, other than the $F W H M$ of $\mathrm{H} \beta$ (apart from the one with $\Gamma_{\mathrm{h}}$ discussed above). In other words, we do not have any indication that the strength of the soft excess may be related to any other important parameter, apart from the FWHM of $\mathrm{H} \beta$, not even the accretion rate.

The "classical" interpretation of the soft excess as direct emission from the accretion disc was questioned when several studies (e.g. Gierliński \& Done 2004; Crummy et al. 2006) showed that the observed temperature of the resulting black body is remarkably constant across orders of magnitude of luminosities and $\mathrm{BH}$ masses. We confirmed the existence of a "universal" temperature, independent of luminosity or BH mass, in the large number of sources of CAIXA, if we substitute in the baseline model the soft X-ray power-law with a thermal component (B09). In the last few years, two main competing models have been proposed to explain the origin of the soft excess, both ascribing its apparent universal temperature to atomic physics processes. In one scenario, the soft excess arises from the enhancement of reflection from the inner regions of the accretion disc due to light-bending effects, together with a strong suppression of the primary emission (e.g. Miniutti \& Fabian 2004). On the other hand, the soft excess can also be mimicked by absorption from a relativistically outflowing warm gas (e.g. Gierliński \& Done 2004). Regardless of the details of the two models (and see also Merloni et al. 2006b, for a self-consistent model of X-ray emission from inhomogeneous, clumpy discs), they both imply that the observed X-ray emission is suppressed with respect to the intrinsic one. 
Table 1. Correlations between the main parameters which characterise the sources in CAIXA.

\begin{tabular}{|c|c|c|c|c|c|c|c|c|c|c|c|c|}
\hline & $\bar{L} L_{2-10}$ & 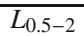 & 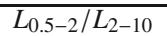 & $E W_{6.4}$ & $\Gamma_{h}$ & $L_{\mathrm{bol}} / L_{\mathrm{Edd}}$ & $\mathrm{H}_{\beta}$ & $M_{\mathrm{BH}}$ & $L_{6 \mathrm{~cm}}$ & $\bar{L} L_{20 \mathrm{~cm}}$ & $R_{\mathrm{X}}$ & $R_{1}$ \\
\hline$L_{2-10}$ & - & $\begin{array}{c}0.92 \\
<-38\end{array}$ & $\mathrm{X}$ & $\begin{array}{c}-0.33 \\
-4.4\end{array}$ & $\mathrm{X}$ & $\mathrm{X}$ & $\begin{array}{c}0.37 \\
-4\end{array}$ & $\begin{array}{c}0.80 \\
-20.1\end{array}$ & $\begin{array}{c}0.65 \\
-11.7\end{array}$ & $\begin{array}{c}0.79 \\
-23.0\end{array}$ & $\mathrm{X}$ & $X$ \\
\hline$L_{0.5-2}$ & $\begin{array}{c}0.92 \\
<-38\end{array}$ & - & $\mathrm{X}$ & $\begin{array}{c}-0.30 \\
-3.2\end{array}$ & $\mathrm{X}$ & $\mathrm{X}$ & $\mathrm{X}$ & $\begin{array}{c}0.67 \\
-10.7\end{array}$ & $\begin{array}{c}0.63 \\
-10.7\end{array}$ & $\begin{array}{c}0.77 \\
-23.1\end{array}$ & X & $\mathrm{X}$ \\
\hline$L_{0.5-2} / L_{2-10}$ & $\mathrm{X}$ & X & - & $\mathrm{X}$ & $\begin{array}{l}0.42 \\
-7.3\end{array}$ & $\mathrm{X}$ & $\begin{array}{l}-0.64 \\
-11.2\end{array}$ & $\mathrm{X}$ & $\mathrm{X}$ & X & X & $\mathrm{X}$ \\
\hline$E W_{6.4}$ & $\begin{array}{c}-0.33 \\
-4.4\end{array}$ & $\begin{array}{c}-0.30 \\
-3.2\end{array}$ & $\mathrm{X}$ & - & $\mathrm{X}$ & $\begin{array}{l}-0.38 \\
-3.2\end{array}$ & $\mathrm{X}$ & $\mathrm{X}$ & $\mathrm{X}$ & $\mathrm{X}$ & $\mathrm{X}$ & $\mathrm{X}$ \\
\hline$\Gamma_{h}$ & $\mathrm{X}$ & $\mathrm{X}$ & $\begin{array}{l}0.42 \\
-7.3\end{array}$ & $\mathrm{X}$ & - & $\mathrm{X}$ & $\begin{array}{c}-0.39 \\
-4.1\end{array}$ & $\mathrm{X}$ & $\mathrm{X}$ & $\mathrm{X}$ & $\mathrm{X}$ & $\mathrm{X}$ \\
\hline$L_{\mathrm{bol}} / L_{\mathrm{Edd}}$ & $\mathrm{X}$ & $X$ & $\mathrm{X}$ & $\begin{array}{l}-0.38 \\
-3.2\end{array}$ & $X$ & - & $\begin{array}{c}-0.40 \\
-3.7\end{array}$ & $X$ & $X$ & $X$ & X & X \\
\hline $\mathrm{H}_{\beta}$ & $\begin{array}{c}0.37 \\
-4\end{array}$ & $\mathrm{X}$ & $\begin{array}{l}-0.64 \\
-11.2\end{array}$ & $\mathrm{X}$ & $\begin{array}{c}-0.39 \\
-4.1\end{array}$ & $\begin{array}{l}-0.40 \\
-3.7\end{array}$ & - & $\begin{array}{c}0.69 \\
-12.0\end{array}$ & $\mathrm{X}$ & $\mathrm{X}$ & $\mathrm{X}$ & $\mathrm{X}$ \\
\hline $\mathrm{M}_{\mathrm{BH}}$ & $\begin{array}{c}0.80 \\
-20.1\end{array}$ & $\begin{array}{c}0.67 \\
-10.7\end{array}$ & $X$ & $\mathrm{X}$ & $\mathrm{X}$ & $\mathrm{X}$ & $\begin{array}{c}0.69 \\
-12.0\end{array}$ & - & $\begin{array}{l}0.49 \\
-4.7\end{array}$ & $\begin{array}{l}0.66 \\
-8.0\end{array}$ & $\mathrm{X}$ & $\mathrm{X}$ \\
\hline$L_{6 \mathrm{~cm}}$ & $\begin{array}{c}0.65 \\
-11.7\end{array}$ & $\begin{array}{c}0.63 \\
-10.7\end{array}$ & $X$ & $\mathrm{X}$ & $\mathrm{X}$ & $\mathrm{X}$ & $\mathrm{X}$ & $\begin{array}{l}0.49 \\
-4.7\end{array}$ & - & $\begin{array}{c}0.92 \\
-25.3\end{array}$ & $\begin{array}{c}0.65 \\
-11.4\end{array}$ & $\begin{array}{l}0.35 \\
-3.2\end{array}$ \\
\hline$L_{20 \mathrm{~cm}}$ & $\begin{array}{c}0.79 \\
-23.0\end{array}$ & $\begin{array}{c}0.77 \\
-23.1\end{array}$ & $\mathrm{X}$ & $X$ & $\mathrm{X}$ & $\mathrm{X}$ & $\mathrm{X}$ & $\begin{array}{l}0.66 \\
-8.0\end{array}$ & $\begin{array}{c}0.92 \\
-25.3\end{array}$ & - & $\begin{array}{l}0.55 \\
-9.5\end{array}$ & $\mathrm{X}$ \\
\hline$R_{\mathrm{X}}$ & $\mathrm{X}$ & $\mathrm{X}$ & $\mathrm{X}$ & $\mathrm{X}$ & $\mathrm{X}$ & $\mathrm{X}$ & $\mathrm{X}$ & $\mathrm{X}$ & $\begin{array}{c}0.65 \\
-11.4\end{array}$ & $\begin{array}{l}0.55 \\
-9.5\end{array}$ & - & $\begin{array}{c}0.58 \\
-14.0\end{array}$ \\
\hline$R_{\mathrm{l}}$ & $\mathrm{X}$ & $\mathrm{X}$ & $\mathrm{X}$ & $\mathrm{X}$ & $\mathrm{X}$ & $\mathrm{X}$ & $\mathrm{X}$ & $\mathrm{X}$ & $\begin{array}{l}0.35 \\
-3.2\end{array}$ & $\mathrm{X}$ & $\begin{array}{c}0.58 \\
-14.0\end{array}$ & - \\
\hline
\end{tabular}

For each pair, the Spearman correlation coefficient $\rho$ is reported (top), along with the logarithm of the NHP (bottom). If the latter is larger than -3.0 , we consider the correlation with low significance and an " $\mathrm{X}$ " is shown. Parameters with no significant correlations, namely $E W_{6.7}, E W_{6.97}$ and $\Gamma_{s}$, are not listed in the table. Correlations involving $L_{0.5-2}$ or $L_{0.5-2} / L_{2-10}$ were performed with the unobscured sub-catalogue of CAIXA. In this table, we also show trivial correlations for completeness, such as the $L_{0.5-2} / L_{2-10}$ versus $\Gamma_{h}$, or the $\mathrm{H} \beta F W H M$ versus $L_{\text {bol }} / L_{\mathrm{Edd}}$ one. See B09 and text for details.

Therefore, the two models predict that the soft excess should be more prominent in X-ray weaker sources. As we have already mentioned, there is no significant correlation between the $\mathrm{X}$-ray luminosity ratio (our proxy for the strength of the soft excess) and any other parameter, apart from the $F W H M$ of the $\mathrm{H} \beta$. We can only look for indications that the latter is correlated to any observed suppression of the intrinsic X-ray continuum. Indeed, we do find a significant correlation between the hard $\mathrm{X}$ ray luminosity and the $F W H M$ of $\mathrm{H} \beta$ (see Table 1 and Fig. 2), confirming previous claims (e.g. Piconcelli et al. 2005). And, more interestingly, we also find that the ratio between the optical flux in the $\mathrm{V}$ band and the hard X-ray flux is significantly anti-correlated $\left(\rho=-0.36-\mathrm{NHP}=2 \times 10^{-4}\right)$ with the width of the $\mathrm{H} \beta$. This clearly shows that the correlation of $L_{0.5-2} / L_{2-10}$ ratio with the $\mathrm{H} \beta F W H M$ is driven by the hard X-ray luminosity becoming weaker, rather than the soft X-ray luminosity becoming stronger. Therefore, there are observational indications that objects with narrower $\mathrm{H} \beta$ are X-ray weaker than normal broadline sources. However, it is impossible from these results to distinguish between an absorption or reflection origin of the soft excess. Only detailed modeling of broadband spectra (including high SNR data above $10 \mathrm{keV}$ ) can solve the issue (e.g. Ponti et al. 2008).

\subsection{Correlations with $\mathrm{BH}$ mass and the $\mathrm{X}$-ray bolometric correction}

The strongest correlation with the BH mass in CAIXA is the one with the hard X-ray luminosity (see Table 1 and Fig. 3). This correlation is expected, because, if the accretion rate is a universal constant, the bolometric luminosity should rise linearly with the $\mathrm{BH}$ mass. Assuming that the bolometric correction, i.e. the ratio between the bolometric and the X-ray luminosity, is constant (as in Elvis et al. 1994), the correlation should be also linear with $\mathrm{X}$-ray luminosity. The best fit slope for our data is slightly lower than $1,0.89 \pm 0.07$, in agreement with previous results (Wang et al. 2009). This flatter slope could suggest that objects with larger BH masses have intrinsically lower accretion rates or are simply X-ray weaker with respect to their bolometric luminosity. On the other hand, the correlation with the radio luminosity $\left(\rho=0.59-\mathrm{NHP}=2 \times 10^{-9}\right.$ for the $20 \mathrm{~cm}$ catalogue $)$ is consistent with a linear relation with the $\mathrm{BH}$ mass (see Fig. 3), thus supporting the hypothesis that the total power of the AGN scales linearly with the $\mathrm{BH}$ mass, while high-luminosity objects may need a larger X-ray bolometric correction factor.

The increasing optical-to-X-ray ratio with increasing optical luminosity is a well established result in AGN research (see e.g. Avni \& Tananbaum 1986; Just et al. 2007, and references therein). This is in agreement with the X-ray bolometric correction factor increasing with luminosity adopted in CAIXA, after Marconi et al. (2004). However, the correlation between the $\mathrm{BH}$ mass and the bolometric luminosity calculated in this way $\left(\rho=0.80-\mathrm{NHP}=7 \times 10^{-21}\right)$ gives a slope steeper than 1 , suggesting that the luminosity dependence of this correction factor may be too strong at large luminosities. Recently, Vasudevan \& Fabian (2009) have suggested that the X-ray bolometric correction may be dependent on the Eddington ratio, not the luminosity. We tried to adopt the average bolometric correction reported in their paper for each Eddington ratio bin. The result is shown in the bottom right plot of Fig. 3: a correlation between the bolometric corrected X-ray luminosity and the BH mass with a slope consistent with 1 is recovered.

Two other significant correlations with $\mathrm{BH}$ mass are found in CAIXA. The first is the correlation between the $\mathrm{BH}$ mass and $z$ (see Fig. 4). This is expected: if the efficiency of the conversion of gravitational energy into radiative output is the same for all AGN, the objects at high $z$, to be in CAIXA, must be luminous and, likely, with a large $\mathrm{BH}$ mass. Note that, at each redshift, narrow-line objects invariably have the smallest BH masses: this is well known and some authors believe that the masses in these 

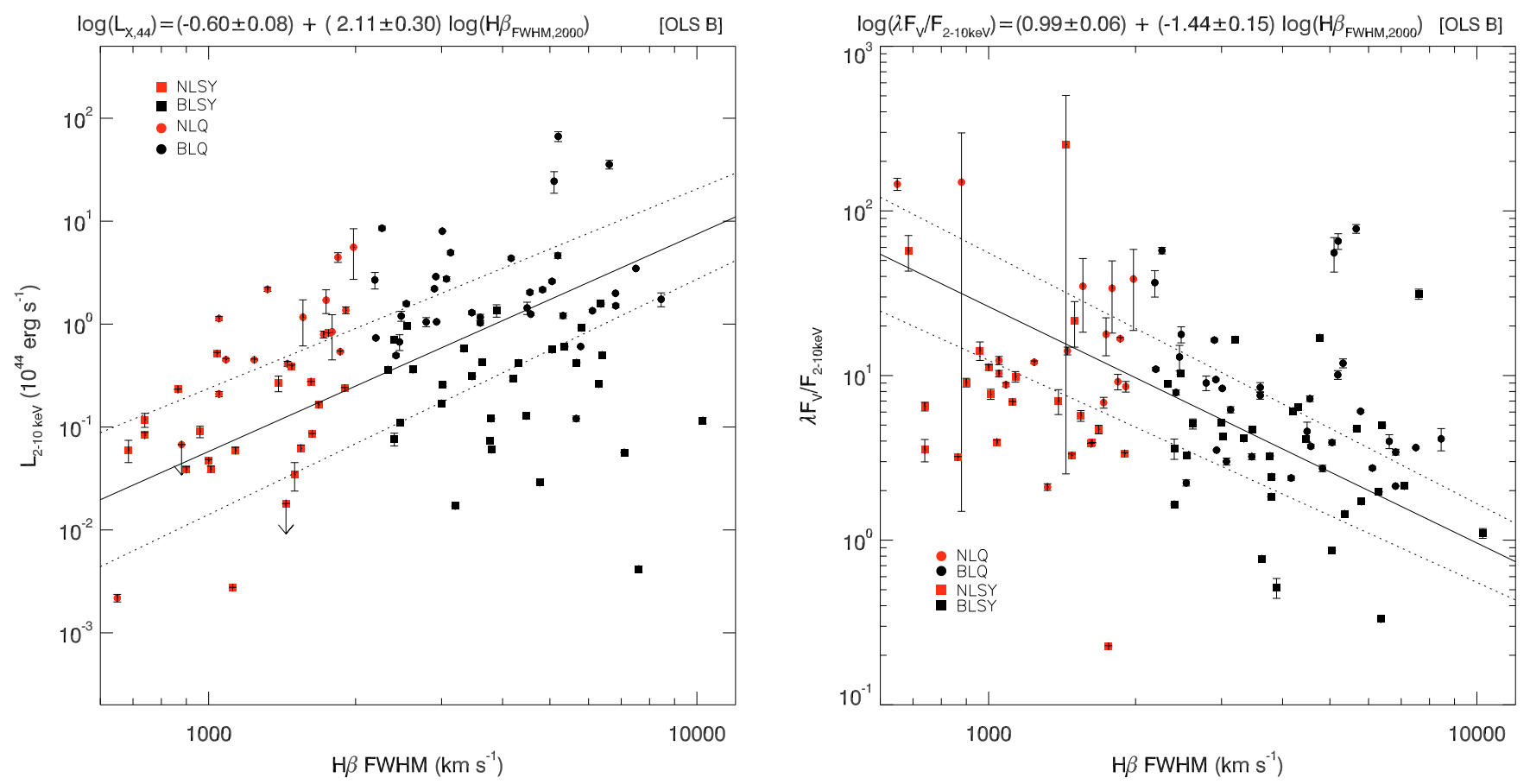

Fig. 2. Left panel: hard X-ray luminosity vs. $F W H M$ of $\mathrm{H} \beta$ : the two parameters appear correlated. Right panel: ratio between the optical flux ( $V$ filter) and the hard X-ray flux against the $F W H M$ of $\mathrm{H} \beta$ : the observed anti-correlation suggests that narrow-line objects may be X-ray underluminous. See text for details and caption of Fig. 5 for details on the adopted symbols.

objects are underestimated (e.g. Decarli et al. 2008; Marconi et al. 2008). This effect represents the other correlation with the $\mathrm{BH}$ mass found in CAIXA and can be explained in terms of the dependence of the $\mathrm{BH}$ mass on the $\mathrm{H} \beta F W H M$. This is of course due to the fact that most of the BH masses in CAIXA were primarily estimated from the $\mathrm{H} \beta F W H M$ under the assumption of a virialised Broad Line Region (BLR). This would imply $M_{\mathrm{BH}} \propto F W H M(\mathrm{H} \beta)^{2}$. The fact that the actual correlation in CAIXA requires a slightly steeper slope $(2.96 \pm 0.23)$ is likely the result of the inhomogeneous $\mathrm{H} \beta$ data and methods for estimation of the BH masses.

No other significant correlations with $\mathrm{BH}$ mass are found in CAIXA. In particular, the correlation with the Eddington ratio is lower than our threshold, even if larger than the $99 \%$ confidence level $\left(\rho=-0.29-\mathrm{NHP}=6 \times 10^{-3}\right)$.

\subsection{Correlations with radio emission and its origin in radio-quiet $A G N$}

In this section, we discuss the correlations between the radio properties of the objects in CAIXA and other parameters. It should be borne in mind that the catalogue does not include radio-loud objects, as explained in detail in B09.

In Fig. 5 (right panels), we plot the hard X-ray versus the radio luminosities (at 6 and $20 \mathrm{~cm}$ ) for the objects in our catalogue. The correlation is highly significant at both wavelengths (see Table 1), with the following best fits:

$$
\begin{aligned}
& \log \left(L_{6,40}\right)=(-0.48 \pm 0.08)+(1.19 \pm 0.08) \log \left(L_{2-10,44}\right) \\
& \log \left(L_{20,40}\right)=(-0.63 \pm 0.06)+(1.13 \pm 0.06) \log \left(L_{2-10,44}\right)
\end{aligned}
$$

where radio and X-ray luminosities are given in units of $10^{40}$ and $10^{44} \mathrm{erg} \mathrm{s}^{-1}$, respectively.

While the radio emission of radio-loud objects has been clearly established as originating in their relativistic jets (e.g. Begelman et al. 1984), the radio activity in radio-quiet sources is still puzzling. Several authors have shown that X-ray and radio luminosity strongly correlate in radio-quiet AGN (e.g. Brinkmann et al. 2000; Panessa et al. 2007; Li et al. 2008; Laor \& Behar 2008). This result is generally interpreted as evidence that also in radio-quiet objects radio emission can be considered a direct tracer of the nuclear activity, either through a common accretion process or through the scale-invariant dependence of the radio-emitting jet on the accretion physics (e.g. Heinz \& Sunyaev 2003; Merloni et al. 2003).

The presence of the strong correlation found in CAIXA is in agreement with previous results, even if the slope of our best fits (around 1.1-1.2) is somewhat steeper than the linear relations found by Brinkmann et al. (2000), Panessa et al. (2007) and Laor \& Behar (2008) for radio-quiet objects. Our slope remains steeper even if we consider the correlation between the radio emission and the soft $\mathrm{X}$-ray luminosity (see Table 1 and Fig. 6), as the above-mentioned authors do (with the exception of Panessa et al. 2007). Other authors found correlations that are far from linear, and that are significantly different between radioloud and radio-quiet objects (e.g. Li et al. 2008). We therefore decided to investigate possible biases affecting this correlation.

It is known that care must be taken in assessing the real significance of any luminosity-luminosity correlation and in giving a physical meaning to the best fit slope, because of the effects introduced by distance and the selection of the catalogue. Indeed, as expected, the luminosity is tightly correlated to the redshift, due to the sensitivity limit of our observations (although it varies depending on the exposure time of each observation).

In the left panels of Fig. 5, we show the same correlations as above, but with fluxes instead of luminosity. The correlations are still statistically significant, but the significance is lower $\left(\rho=0.49\right.$ and 0.67 , with NHP $=7 \times 10^{-7}$ and $5 \times 10^{-16}$, for $F_{6}$ and $F_{20}$, respectively) and the scatter is much larger than that between luminosities. Moreover, the best slopes are flatter than the 

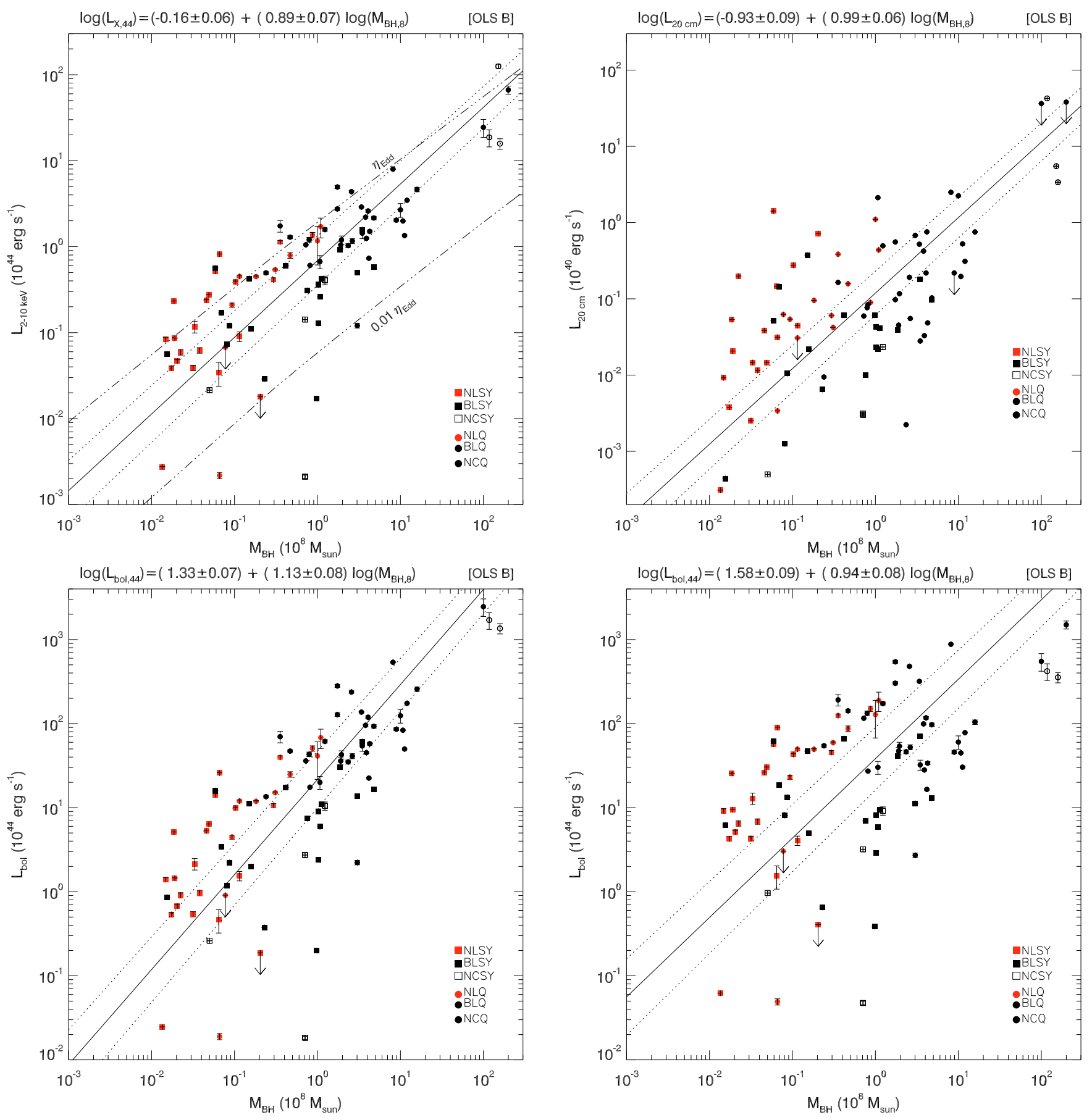

Fig. 3. Top 2-10 keV X-ray and $20 \mathrm{~cm}$ radio luminosity vs. BH mass for all the sources in the catalogue with an estimate of the latter. A linear regression fit is superimposed on the plots. In the left panel, the curves referring to Eddington rates of 1 and 0.01 are also shown. Bottom Bolometric luminosity (left: luminosity-dependent (Marconi et al. 2004); right: eddington ratio-dependent (Vasudevan \& Fabian 2009)) vs. BH mass for all the sources in the catalogue with an estimate of the latter. A linear regression fit is superimposed on the plots. See text for details.

ones found with luminosities, in particular for the measures at $20 \mathrm{~cm}$, where there are many upper limits. This suggests that the role of distance in the previous luminosity-luminosity plots is indeed important. The reason is obvious: only objects with high luminosities in both bands can be seen at large distances, while both high-and-low luminosity objects can be seen at small distances (but the small volume often reduces the number of high$\mathrm{L}$ objects). This was shown in the simulations by Isobe et al. (1986), who demonstrated that, if the upper limits are maintained, then survival analysis methods can in principle recover the correct relationship. However, our catalogue can still be affected by this effect, because, even if we keep the upper limits (both on radio and X-ray fluxes), neither the X-ray nor the radio datasets are by any means complete and they are selected only on the basis of the SNR of the first, thus likely introducing a bias towards high flux sources. We therefore do not consider the existence of a significant correlation between fluxes a significant enough proof that the distance bias does not play a role in the luminosity-luminosity correlation in CAIXA. 


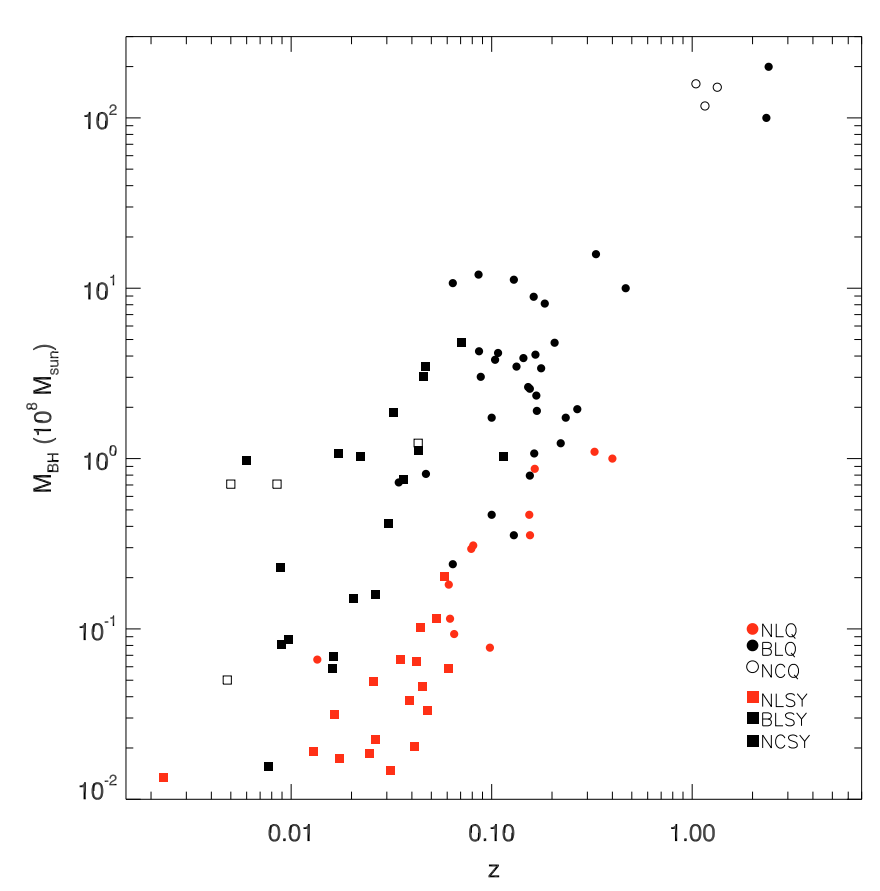

Fig. 4. BH mass vs redshift. See text for details and caption of Fig. 5 for details on the adopted symbols.

Accordingly, we performed a partial Kendall $\tau$ correlation test on censored data, using the distance as the third variable, as proposed by Akritas \& Siebert (1996). Also taking into account the correlation with distance, the X-ray versus radio luminosity correlation still remains significant in all the cases: coefficient $\tau=0.24$ and variance $\sigma=0.056\left(\mathrm{NHP}=3 \times 10^{-5}\right)$ for $6 \mathrm{~cm}$, $\tau=0.22$ and $\sigma=0.046\left(\mathrm{NHP}=1 \times 10^{-6}\right)$ for $20 \mathrm{~cm}$.

We finally performed the "scrambling test" (e.g. Bregman 2005; Merloni et al. 2006a). The principle of this test is very simple: we keep together each pair of X-ray luminosity and redshift and "scramble" the radio fluxes, assigning each one to a random $L_{2-10} / z$ pair. Then, adopting the newly assigned redshift, we calculate the radio luminosity from the random flux and correlate it to the assigned X-ray luminosity. Any physical correlation intrinsic to the original dataset must disappear in the new artificial datasets, because the radio flux of a given object is now associated with the X-ray luminosity of another one. We produced 100000 scrambled datasets with this procedure for the 6 and the $20 \mathrm{~cm}$ radio fluxes and we calculated for each of them the Spearman correlation coefficient, the Kendall partial correlation coefficient and the censored fit. The results are shown in Fig. 7.

It is striking to see how the Spearman correlation coefficients for the real datasets are well within the distribution of the simulated ones: in the case of the $6 \mathrm{~cm}$ data, around 87000 values of $\rho$ are actually larger than the "real" one. Therefore, this test suggests that the probability that the original correlation is driven entirely by distance is very high, i.e. $87 \%$. This probability is much lower for the $20 \mathrm{~cm}$ data (which includes upper limits), but still large: $8 \%$. The simulated distributions of the Kendall partial correlation coefficients are instead shifted towards less significant values with respect to the real ones, but still the probability that distance plays the main role is rather high, being 7 and $4 \%$, for 6 and $20 \mathrm{~cm}$, respectively. However, in Fig. 8 we also show the distributions of the best fit slopes for the simulated datasets compared to the "real" ones (right plots): the latter are much flatter. This indicates that the observed slopes are not random, and may still reflect a real intrinsic correlation. On the other hand, this effect may also be due to the fact that CAIXA contains few objects with large redshifts and no radio fluxes below a certain limit. These two factors contribute to enhance the population of very luminous radio objects in the simulated datasets (when an average or large radio flux is associated with a large $z$ ), while the region with high redshift and low radio luminosity is still under-populated, leading to steeper slopes, i.e. objects more radio-luminous than the real ones.

As a last check, we also performed the "scrambling test" for the flux-flux correlations. In this case, the simulated Spearman correlation coefficients are centered (both for 6 and $20 \mathrm{~cm}$ ) at 0 , as expected for random uncorrelated points (see Fig. 10). Not even a simulated dataset has a correlation coefficient as large as the ones measured in the real datasets, in agreement with the very low estimated NHP in the latter. However, the strong correlation found between the X-ray and radio fluxes in CAIXA may still be implicitly due by distance effects. Due to selection effects, X-ray fluxes are tightly linked to redshift. This is clearly shown in Fig. 9, where we plot the redshift distributions of the sources in CAIXA in four hard X-ray flux bins. The evolution of the distributions is evident: faint objects are clustered at high $z$, while bright sources are peaked at low $z$. Therefore, although we cannot exclude the presence of a physical correlation between hard X-ray and radio fluxes in CAIXA, we are unable to disentangle it from the selection effects present in the catalogue.

In conclusion, the observed correlation between radio and hard X-ray luminosity appears extremely significant on the basis of standard statistical tests (Spearman correlation coefficients, partial Kendall correlation), but the role of the distance effect may be dominant in determining the significance and functional relation. In particular, the scrambling test shows that even the widely used partial Kendall correlation may underestimate the distance effects, thus care must be taken when interpreting the results derived from it. However, random scrambling cannot reproduce the observed slope of the luminosity correlation, and destroys the flux-flux correlation, which is instead observed to be significant. Both results may represent signs of a real underlying correlation, but they also could be induced by the incompleteness of CAIXA.

Therefore, although a physical correlation may be present in our data, we conservatively cannot draw any conclusion from the X-ray/radio correlations in CAIXA. We would like to stress that this is true for CAIXA, which includes upper limits, but it is by no means a flux-limited or volume-limited sample, while distance effects may of course play less important roles in other catalogues or in complete samples.

In the left panel of Fig. 11, we plot the correlation between the two radio-loudness parameters used to exclude the radioloud objects in CAIXA, one based on the optical fluxes found in the literature, the other on the X-ray fluxes measured in this work (see B09 for details). As expected, a highly significant linear regression between the two parameters is found (see Table 1). The slope of the best fit $(0.88 \pm 0.06)$ is in agreement with the ones found by Terashima \& Wilson (2003) and Panessa et al. (2007), even if their samples also include radio-loud objects, thus spanning a larger range both for $R_{\mathrm{X}}$ and $R_{\mathrm{O}}$.

In the right panel of Fig. 11, we show the X-ray radioloudness parameter versus the Eddington ratio. Ho (2002) found an highly significant anti-correlation between these two parameters, suggesting that weakly active nuclei are powered by advection-dominated accretion flows (ADAFs), which give rise to radio-loud spectra. Although this trend seems to be observed in CAIXA, the anti-correlation is not significant $(\rho=-0.14-$ $\mathrm{NHP}=0.19$ ). However, this is not unexpected, because our catalogue does not include radio-loud objects and, indeed, the large 

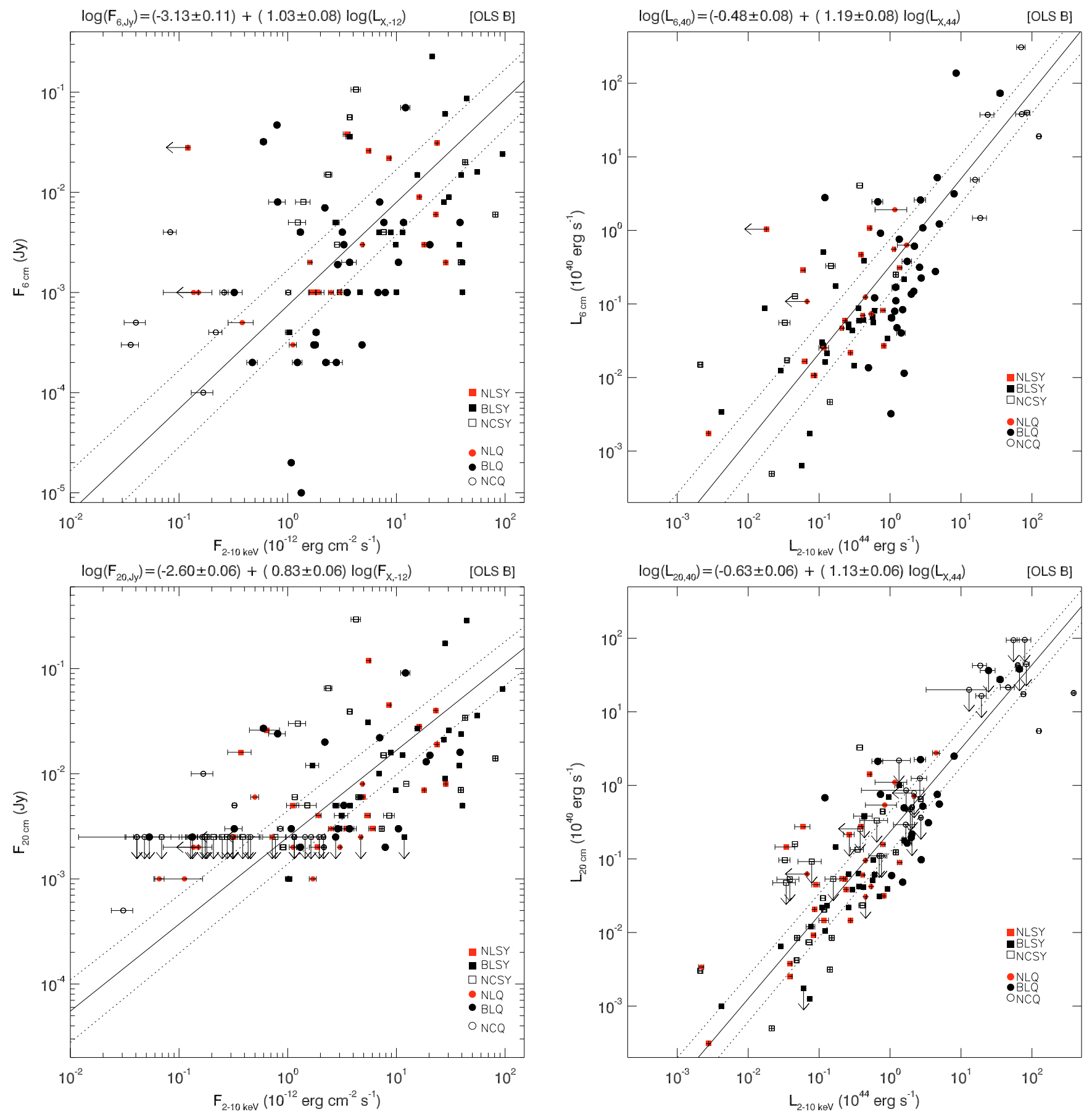

Fig. 5. Radio to X-rays plots. From top to bottom: $2-10 \mathrm{keV}$ vs. $6 \mathrm{~cm}$ emission, $2-10 \mathrm{keV}$ vs. $20 \mathrm{~cm}$ emission. Flux-flux correlations are on the left, luminosity-luminosity correlations on the right. In each case, the analytical expression for the best fit is on the top. The different symbols refers to the classification of the objects, on the basis of their absolute magnitude and $\mathrm{H} \beta$ FWHM: NLSY, narrow-line Seyfert 1; BLSY, broad-line Seyfert 1; $N C S Y$, not-classified Seyfert 1 (no $\mathrm{H} \beta F W H M$ measure available); $N L Q$, narrow-line quasar; $B L Q$, broad-line quasar; $N C Q$, not-classified quasar (no $\mathrm{H} \beta F W H M$ measure available).

$R_{\mathrm{X}}$-low $L_{\mathrm{bol}} / L_{\mathrm{Edd}}$ region is not populated in our plot. Finally, we note here that no correlation is apparent between the soft to hard $\mathrm{X}$-ray luminosity ratio and the radio luminosity.

\subsection{The $X$-ray spectral index}

Apart from the correlations with $\mathrm{H} \beta$ and the X-ray luminosity ratio, already discussed in Sect. 2.1, there are no other significant correlations with the hard X-ray spectral index in CAIXA. In particular, in the literature there are claims of a correlation between $\Gamma_{h}$ and the $2-10 \mathrm{keV}$ luminosity (e.g. Dai et al. 2004; Saez et al. 2008), but there are also several studies that do not find such a correlation (e.g. Reeves \& Turner 2000; George et al. 2000; Winter et al. 2009). In CAIXA, this correlation is not significant ( $96 \%$ confidence level): we note here that this confidence level is not far from the ones quoted by Dai et al. (2004) and Saez et al. (2008), unless the correlation is calculated in particular bins of (relatively high) redshift. In other words, the low significance of the $\Gamma_{h} / L_{2-10}$ correlation in CAIXA is in agreement with previous studies, even with those which claim 


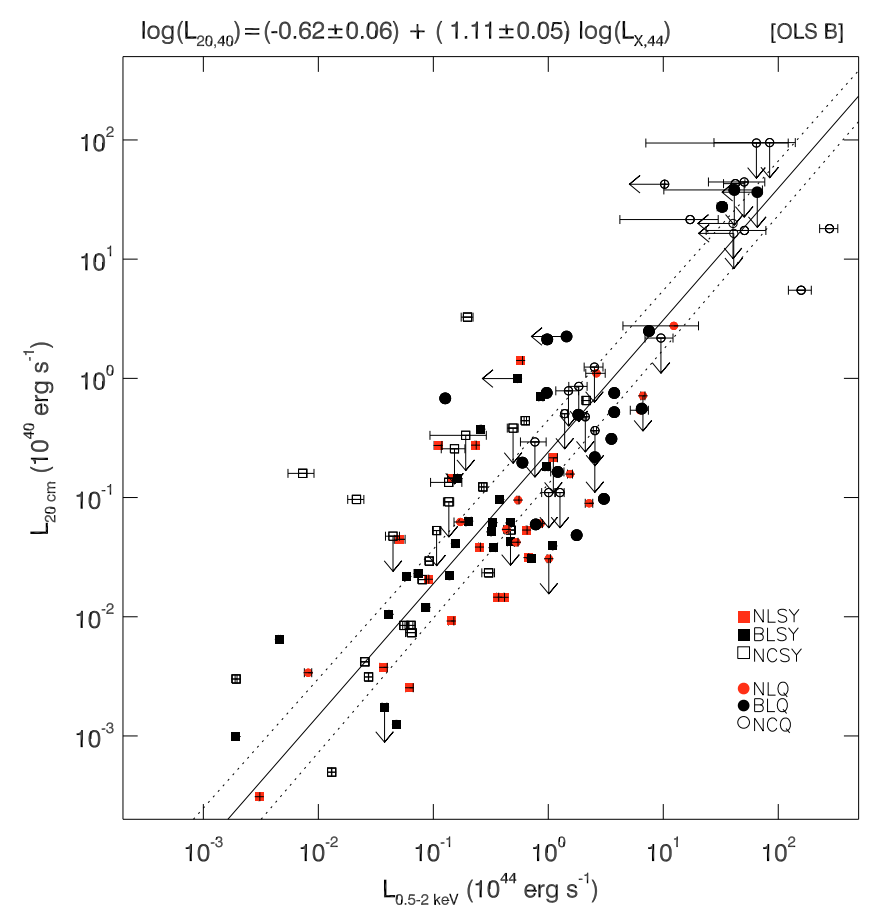

Fig. 6. Radio $20 \mathrm{~cm}$ vs. soft X-ray luminosity, along with best fit. See caption of Fig. 5 for details on the adopted symbols.
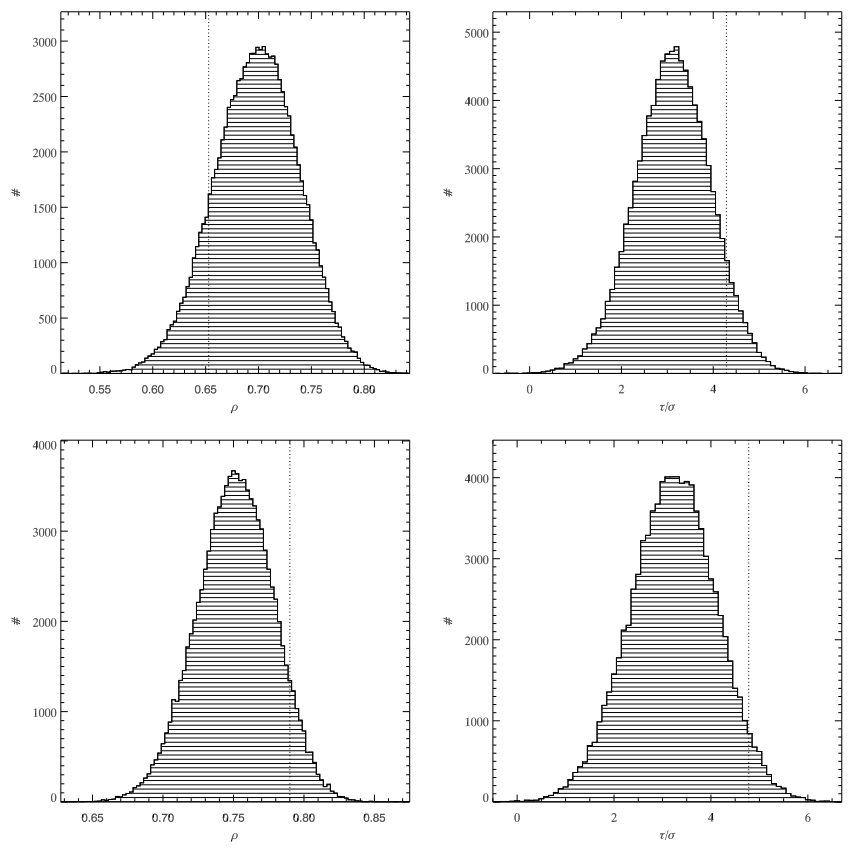

Fig. 7. Scrambling test results for the correlations between the hard $\mathrm{X}$-ray luminosity and the radio luminosity at $6 \mathrm{~cm}$ (upper plots) and $20 \mathrm{~cm}$ (lower plots). The left panels show the distribution of the Spearman coefficients for the 100000 simulated datasets, in comparison to the one measured for the real dataset (dashed vertical line). Same in the right panels, but for the Kendall partial correlation coefficient $\tau$ divided by the variance $\sigma$.

the correlation is real. Nonetheless, a significant difference between the spectral index of Seyferts and quasars is measured in CAIXA $\left(\Gamma_{S y}=1.66 \pm 0.05 ; \Gamma_{\text {QSO }}=1.80 \pm 0.05:\right.$ B09 $)$. We showed in B09 that the introduction of a luminosity-dependent Compton reflection component to the baseline model does not remove the difference between these two populations. Further
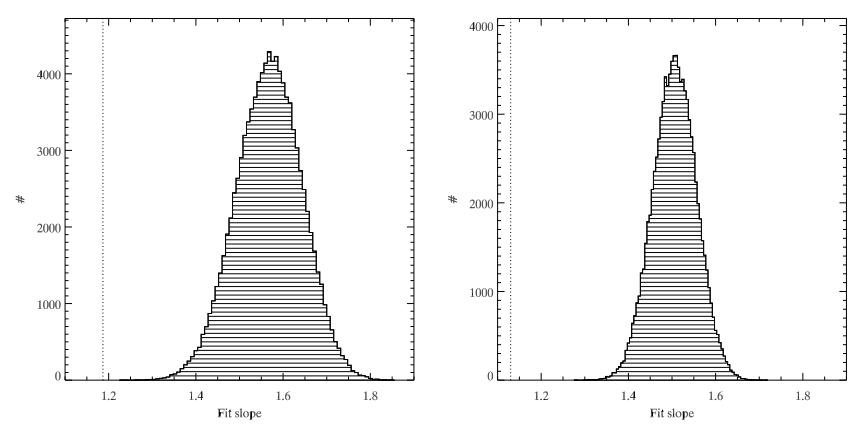

Fig. 8. Scrambling test results for the correlations between the hard $\mathrm{X}$-ray luminosity and the radio luminosity at $6 \mathrm{~cm}$ (left) and $20 \mathrm{~cm}$ (right). The best fit slope distributions for the 100000 simulated datasets are shown, in comparison to the ones measured for the real dataset (dashed vertical line). See text for details.
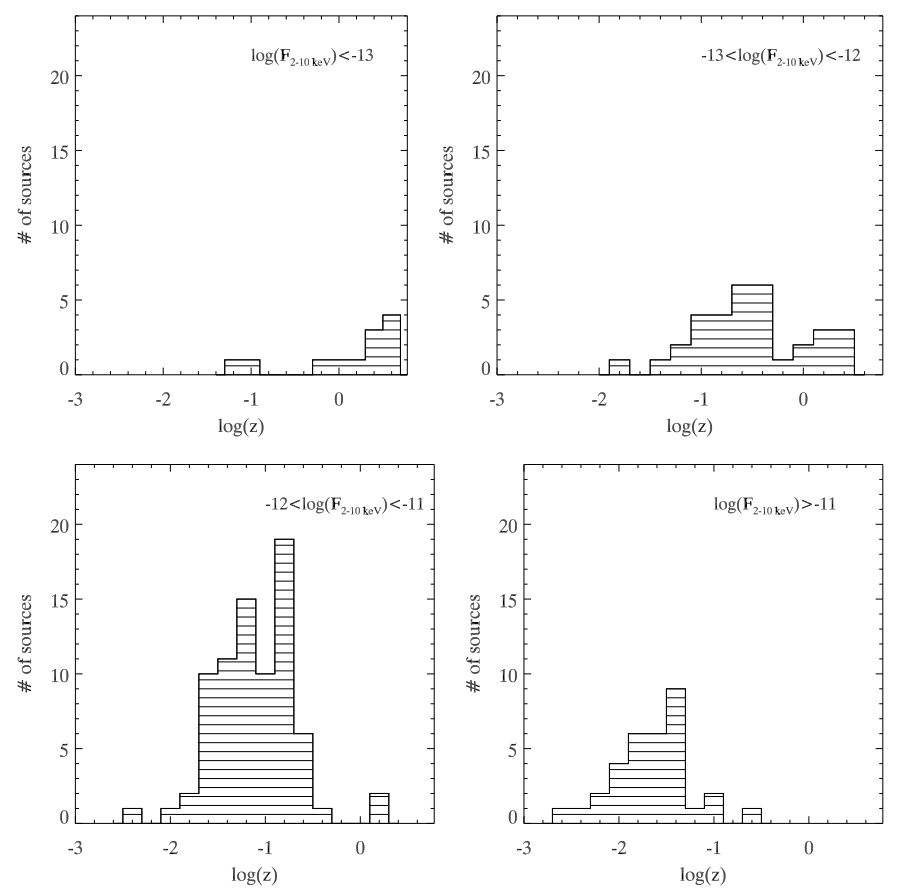

Fig. 9. Redshift distributions of the sources in CAIXA as a function of hard X-ray flux. The distributions clearly evolve with flux, revealing an intrinsic bias of the catalogue, due to selection effects. See text for details.
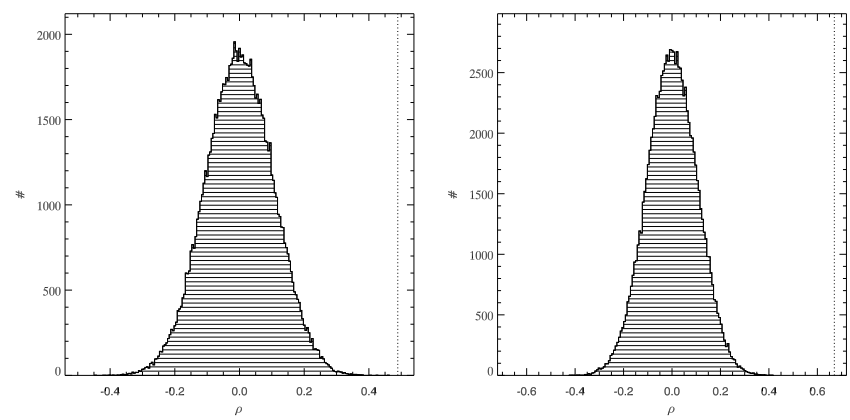

Fig. 10. Scrambling test results for the correlations between the hard $\mathrm{X}$-ray flux and the radio flux at $6 \mathrm{~cm}$ (left) and $20 \mathrm{~cm}$ (right). The distributions of the Spearman coefficients for the 100000 simulated datasets are shown, in comparison to the ones measured for the real dataset (dashed vertical line). 

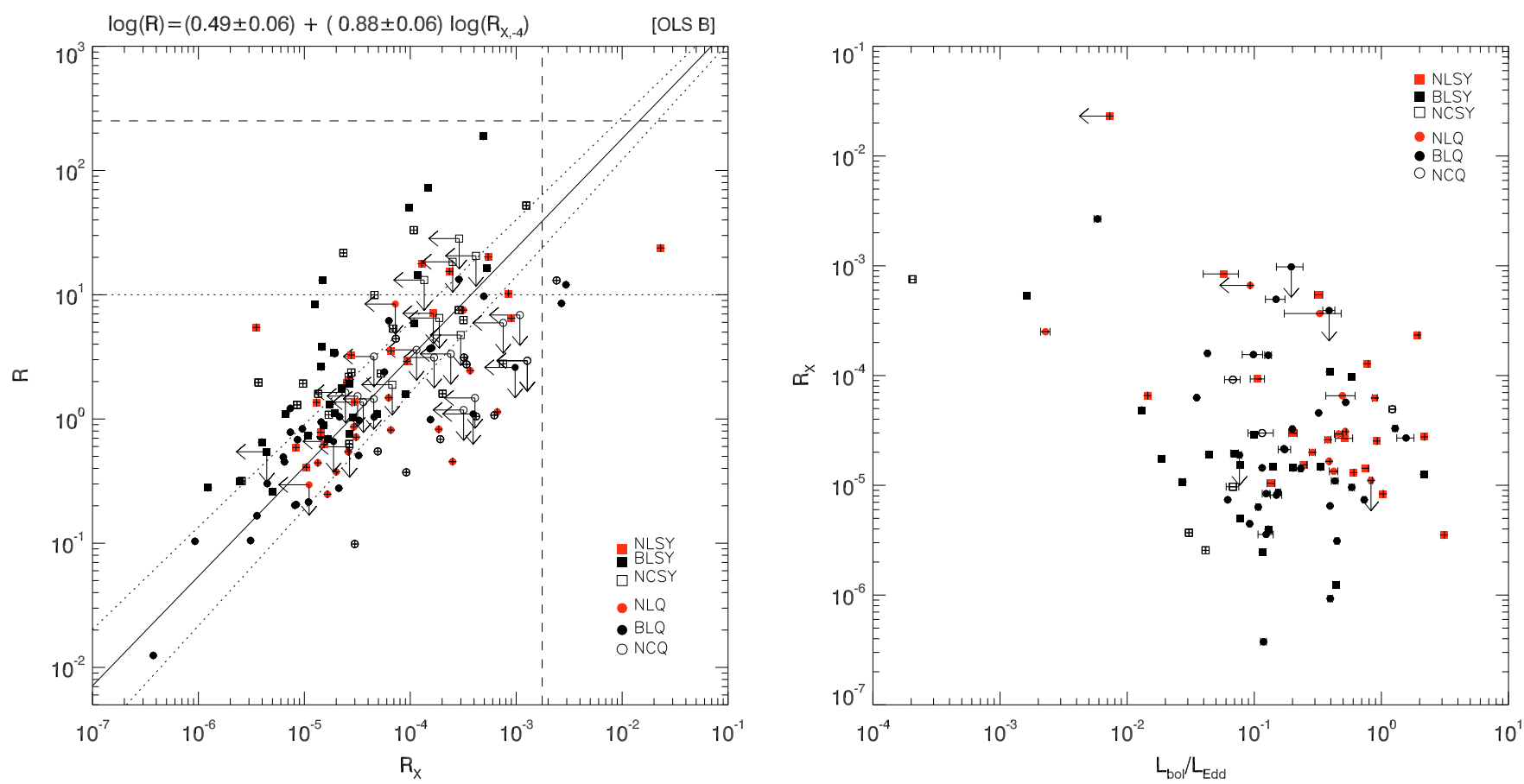

Fig. 11. Left: radio-loudness parameter vs. X-ray radio-loudness parameter (see text for definitions) for all the sources in the catalogue with a radio measure. The dotted horizontal line represents the canonical separation between radio-loud and radio-quiet quasars, while the dashed lines the separation for Seyferts, based both on the optical as well as on the X-ray radio-loudness parameter (after Panessa et al. 2007). A linear regression fit is superimposed on the plot, along with the best fit parameters. We refer the reader to B09 for the detailed selection criteria adopted in CAIXA with respect to the radio-loudness. Right: X-ray radio-loudness parameter against Eddington ratio. Although a trend is observed, the anti-correlation is not statistically significant. See caption of Fig. 5 for details on the adopted symbols.

tests should be performed to understand the real driver of this difference.

Similarly, we do not find a significant correlation between the photon index and the $\mathrm{BH}$ mass or the Eddington rate, but the significances found by Piconcelli et al. (2005) are lower than our threshold, and the results recently found by Kelly et al. (2008) are strongly dependent on how the BH masses are calculated. On the other hand, the correlation with the Eddington ratio found by Shemmer et al. (2008) is more significant than our threshold, but only when high- $z$ and high-luminosity quasars are taken into account.

\subsection{The iron lines}

Bianchi et al. (2007) discussed in detail the strong anticorrelations between the equivalent width $(E W)$ of the neutral iron narrow emission line and the $2-10 \mathrm{keV}$ luminosity and Eddington rate. Despite the exclusion of a source (PMNJ06236436) in CAIXA, the results published in Bianchi et al. (2007) do not suffer from any variation. Recently, Dadina (2008) confirmed the so-called "Iwasawa-Taniguchi" (IT) effect considering the 20-100 keV luminosity, with a slope fully consistent with the one found with CAIXA in the $2-10 \mathrm{keV}$ band.

It is interesting to note that the likely interpretation of the IT effect as due to the covering factor of the torus decreasing with luminosity would imply a decrease of the fraction of Compton-thick objects with luminosity. This is indeed what is found by Della Ceca et al. (2008). From their results, the fraction of Compton-thick objects with respect to the total AGN population, which can be considered a proxy for the covering factor of the torus, can be estimated. We found that it decreases with luminosity following a slope of $\simeq-0.22$, in very good agreement with the IT effect.

We note here that the IT effect is also in agreement with the recent discovery of a non-linear relation between thermal emission from dust and optical luminosity in AGN, which implies a decrease of the covering factor of dust with luminosity, with a slope of $\simeq-0.18$ (Maiolino et al. 2007), i.e. very close to the one which characterises the IT effect. Although the agreement is very good and deserves further investigation, the two methods sample, in principle, different materials. The iron $\mathrm{K} \alpha$ line is mainly produced in Compton-thick gas, while the dust can be associated both with Compton-thin and thick gas.

We do not find any significant correlation involving the $E W \mathrm{~s}$ of the ionised iron lines.

\section{Conclusions}

In this paper, we investigated the correlations between the X-ray and the multiwavelength properties of the sources in CAIXA, a Catalogue of AGN in the XMM-Newton Archive, presented in detail in a companion paper (B09). The main results can be summarised as follows:

- Correlations with $H \beta F W H M$. A very strong anti-correlation between the $F W H M$ of $\mathrm{H} \beta$ and the ratio between the soft and the hard X-ray luminosity is present in CAIXA. In particular, our catalogue does not contain narrow-line objects with a $L_{0.5-2} / L_{2-10}$ ratio lower than 1 . Although this correlation reflects the same physical driver as correlations already published in the literature between the soft X-ray slope and the H $\beta$ FWHM (Boller et al. 1996; Laor et al. 1997), we consider that it has a more fundamental meaning, because it links two 
more model-independent quantities (it is interesting to notice that no correlation between the soft X-ray slope and the $\mathrm{H} \beta F W H M$ is found in CAIXA). The ratio between the optical flux in the $\mathrm{V}$ band and the hard X-ray flux is also significantly anti-correlated with the $\mathrm{H} \beta$ line width, suggesting that objects with narrower $\mathrm{H} \beta$ are $\mathrm{X}$-ray weaker than normal broad-line sources.

- Correlations with the BH mass. The strong correlation between the X-ray luminosity and the $\mathrm{BH}$ mass has a slope flatter than 1, suggesting that high-luminosity objects may be X-ray weaker. A luminosity dependent bolometric factor could explain this result, but the one proposed by Marconi et al. (2004) seems too strong at high luminosities. On the other hand, with the bolometric factor proposed by Vasudevan \& Fabian (2009), which depends on the Eddington ratio, a relation with a slope consistent with 1 is recovered. In general, that the intrinsic total power of AGN indeed scales linearly with $\mathrm{BH}$ mass is suggested by the linear relation between the radio luminosity and the $\mathrm{BH}$ mass.

- Correlations with radio emission. In CAIXA there is a very strong correlation between the radio $(6$ or $20 \mathrm{~cm}$ ) and the X-ray (soft or hard) luminosity. Similar correlations were presented and discussed by several authors in the past (e.g. Brinkmann et al. 2000; Panessa et al. 2007; Li et al. 2008). We have critically reviewed the effect that the incompleteness of the sample may have on this correlation. The "scrambling test" suggests that indeed a distance bias may be dominant, even if the partial Kendall $\tau$ test often used in the literature to account for this bias suggests otherwise. Our conclusion points to the need to use complete unbiased samples, such as that recently discussed by Laor \& Behar (2008), to draw observational constraints on the nature of radio emission in radio-quiet AGN.

Acknowledgements. S.B. and G.M. acknowledge financial support from ASI (grant I/088/06/0), G.P. from ANR (ANR-06-JCJC-0047). We acknowledge support from the Faculty of the European Space Astronomy Centre (ESAC). We thank Andrea Merloni, Eric Feigelson, F. Massaro and G. Miniutti for useful discussions, and Craig Gordon for all his efforts in solving problems related to Xspec. We thank the anonymous referee for valuable suggestions. Based on observations obtained with XMM-Newton, an ESA science mission with instruments and contributions directly funded by ESA Member States and NASA.

\section{Appendix A: Linear fits on censored data}

This appendix is very similar to the one presented in Bianchi et al. (2007). We include it here for the convenience and to report the inclusion of the scattering error in the overall uncertainty estimated for the fit parameters.

Since we are dealing with a large number of upper limits, we followed Guainazzi et al. (2006) to perform a linear fit, taking into account both upper limits and errors on the $y$ parameter. Their method is an extension of the regression method on censored data described by Schmitt (1985) and Isobe et al. (1986) and can be summarized as follows. Several ordinary least squares Y vs. X (OLS Y/X) or bisector (OLS B) fits (each plot reports on the upper right which method was used) was performed on a set of Monte-Carlo simulated data derived from the experimental points according to the following rules: a) each detection was substituted by a random Gaussian distribution, whose mean is the best-fit measurement and whose standard deviation is its statistical uncertainty; b) each upper limit $U$ was substituted by a random uniform distribution in the interval
$[0, U]$. The mean of the slopes and the intercepts derived from the fits of each data set are our "best fit". Note that errors and upper limits on the $x$ parameter are not considered in the procedure, although they are plotted. This does not affect the results significantly, given the fact that they are typically much smaller than the errors on the $y$. Moreover, the errors on the best fit parameters only take into account the experimental uncertainties, the dispersion of these parameters being within the Monte Carlo simulated datasets. Any intrinsic, physical scattering of the data points is not included. To test the significance of this anti-correlation, we calculated the Spearman's rank coefficient for each Monte Carlo simulated dataset; the mean value is taken as the Spearman's rank coefficient of our "best fit".

\section{References}

Akritas, M. G., \& Siebert, J. 1996, MNRAS, 278, 919

Avni, Y., \& Tananbaum, H. 1986, ApJ, 305, 83

Begelman, M. C., Blandford, R. D., \& Rees, M. J. 1984, Rev. Mod. Phys., 56, 255

Bianchi, S., Guainazzi, M., Matt, G., \& Fonseca Bonilla, N. 2007, A\&A, 467, L19

Bianchi, S., Guainazzi, M., Matt, G., Fonseca Bonilla, N., \& Ponti, G. 2009, A\&A, 495, 421

Boller, T., Brandt, W. N., \& Fink, H. 1996, A\&A, 305, 53

Boroson, T. A., \& Green, R. F. 1992, ApJS, 80, 109

Brandt, W. N., Mathur, S., \& Elvis, M. 1997, MNRAS, 285, L25

Bregman, J. N. 2005, ArXiv Astrophysics e-prints

Brinkmann, W., Laurent-Muehleisen, S. A., Voges, W., et al. 2000, A\&A, 356, 445

Crummy, J., Fabian, A. C., Gallo, L., \& Ross, R. R. 2006, MNRAS, 365, 1067 Dadina, M. 2008, A\&A, 485, 417

Dai, X., Chartas, G., Eracleous, M., \& Garmire, G. P. 2004, ApJ, 605, 45 Decarli, R., Dotti, M., Fontana, M., \& Haardt, F. 2008, MNRAS, 386, L15 Della Ceca, R., Caccianiga, A., Severgnini, P., et al. 2008, A\&A, 487, 119 Elvis, M., Wilkes, B. J., McDowell, J. C., et al. 1994, ApJS, 95, 1 George, I. M., Turner, T. J., Yaqoob, T., et al. 2000, ApJ, 531, 52 Gierliński, M., \& Done, C. 2004, MNRAS, 349, L7

Guainazzi, M., Siemiginowska, A., Stanghellini, C., et al. 2006, A\&A, 446, 87 Heinz, S., \& Sunyaev, R. A. 2003, MNRAS, 343, L59

Ho, L. C. 2002, ApJ, 564, 120

Isobe, T., Feigelson, E. D., \& Nelson, P. I. 1986, ApJ, 306, 490

Just, D. W., Brandt, W. N., Shemmer, O., et al. 2007, ApJ, 665, 1004

Kelly, B. C., Bechtold, J., Trump, J. R., Vestergaard, M., \& Siemiginowska, A. 2008, ApJS, 176, 355

Laor, A., \& Behar, E. 2008, MNRAS, 390, 847

Laor, A., Fiore, F., Elvis, M., Wilkes, B. J., \& McDowell, J. C. 1994, ApJ, 435, 611

Laor, A., Fiore, F., Elvis, M., Wilkes, B. J., \& McDowell, J. C. 1997, ApJ, 477, 93

Li, Z.-Y., Wu, X.-B., \& Wang, R. 2008, ApJ, 688, 826

Lynden-Bell, D. 1969, Nature, 223, 690

Maiolino, R., Shemmer, O., Imanishi, M., et al. 2007, A\&A, 468, 979

Marconi, A., Risaliti, G., Gilli, R., et al. 2004, MNRAS, 351, 169

Marconi, A., Axon, D. J., Maiolino, R., et al. 2008, ApJ, 678, 693

Merloni, A., Heinz, S., \& di Matteo, T. 2003, MNRAS, 345, 1057

Merloni, A., Körding, E., Heinz, S., et al. 2006a, New Astron., 11, 567

Merloni, A., Malzac, J., Fabian, A. C., \& Ross, R. R. 2006b, MNRAS, 370, 1699

Miniutti, G., \& Fabian, A. C. 2004, MNRAS, 349, 1435

Panessa, F., Barcons, X., Bassani, L., et al. 2007, A\&A, 467, 519

Piconcelli, E., Jimenez-Bailón, E., Guainazzi, M., et al. 2005, A\&A, 432, 15

Ponti, G., Cappi, M., Genghini, M., et al. 2008, Mem. Soc. Astron. Ital., 79, 274

Reeves, J. N., \& Turner, M. J. L. 2000, MNRAS, 316, 234

Saez, C., Chartas, G., Brandt, W. N., et al. 2008, AJ, 135, 1505

Schmitt, J. H. M. M. 1985, ApJ, 293, 178

Shemmer, O., Brandt, W. N., Netzer, H., Maiolino, R., \& Kaspi, S. 2008, ApJ, 682,81

Terashima, Y., \& Wilson, A. S. 2003, ApJ, 583, 145

Vasudevan, R. V., \& Fabian, A. C. 2009, MNRAS, 392, 1124

Wang, J., Mao, Y. F., \& Wei, J. Y. 2009, AJ, 137, 3388

Winter, L. M., Mushotzky, R. F., Reynolds, C. S., \& Tueller, J. 2009, ApJ, 690, 1322 